\title{
A condition for the uniqueness of Gibbs states in one-dimensional models
}

\author{
Azer Kerimov* \\ Department of Mathematics, Bilkent University, 06533 Bilkent, Ankara, Turkey
}

Received 3 October 1997; received in revised form 27 April 1998

\begin{abstract}
Uniqueness of limit Gibbs states of one dimensional models with a unique "stable" ground state is established at low temperatures. (c) 1998 Elsevier Science B.V. All rights reserved.

Keywords: Hamiltonian; Ground state; Gibbs state; One-dimensional contour; Extreme Gibbs state
\end{abstract}

\section{Introduction}

The problem of phase transitions in one-dimensional models has attracted the interest of many authors during the last decades. The existence (the absence) of phase transitions in some one-dimensional models was proved in [1-13].

In this paper we investigate sufficient conditions for the uniqueness of limit Gibbs states in one-dimensional models.

It is well known that the condition $\sum_{x \in \mathbf{Z}^{1}} x U(x)<\infty(U(x)$ is a pair potential of long range) implies uniqueness of limit Gibbs states [1-3]. In this paper we consider models including the alternative case $U(x) \sim 1 / x^{1+\alpha}$, where $0<\alpha<1$.

In this work we develop a method establishing uniqueness of Gibbs states under very natural conditions similar to the conditions for two- or more-dimensional models.

\section{The main result}

Let us consider a model on $\mathbf{Z}^{1}$ with the Hamiltonian

$$
H(\varphi(x))=\sum_{B \subset \mathbf{Z}^{1}} U(\varphi(B)),
$$

\footnotetext{
*E-mail:kerimov@fen.bilkent.edu.tr.
} 
where the spin variables $\varphi(x) \in \Phi, \Phi$ is a finite set, the potential $U(\varphi(B))$ is not necessarily a translationally invariant function.

On the potential $U(\varphi(B))$ we impose the natural condition which is necessary for the thermodynamic limit:

$$
\sum_{B \subset \mathbf{Z}^{1}: x \in B}|U(\varphi(B))|<\text { const },
$$

where the const does not depend on $x$ and the configuration $\varphi(x)$.

We suppose that model (1) has a unique ground state $\varphi^{g r}(x)$ and satisfies the following stability condition: for any finite set $A \subset \mathbf{Z}^{1}$ with length $|A|$

$$
H\left(\varphi^{\prime}(x)\right)-H\left(\varphi^{g r}(x)\right) \geqslant t|A|,
$$

where $t>0,|A|$ is the number of sites of $A$ and $\varphi^{\prime}(x)$ is a perturbation of the ground state $\varphi^{g r}$ on the finite set $A$.

We also suppose that the potential $U(B)$ satisfies some natural decreasing condition (see Eq. (22)).

Theorem 1. There exists $\beta_{c r}>0$, such that at any $\beta>\beta_{c r}$ the model (1) has a unique limit Gibbs state.

By the uniqueness of Gibbs states we mean the non-existence of two different Gibbs states.

We prove Theorem 1 based on the ideas introduced in [9]. The main idea of the proof is the following.

Let $I_{V}$ be the segment $[-V, V]$. Suppose that the boundary conditions $\varphi(x)=\varphi^{1}(x)$, $x \in \mathbf{Z}^{1}-I_{V}$ are fixed and

$$
H_{V}\left(\varphi(x) \mid \varphi^{1}(x)\right)=\sum_{B \subset \mathbf{Z}^{1}: B \cap[a, b] \neq \emptyset} U(\varphi(B)) .
$$

A set of all configurations $\varphi(x) ; x \in I_{V}$ we denote by $\Phi(V)$.

Due to conditions (2) and (3) the partition function $\Xi_{V}^{1}$ corresponding to the boundary conditions $\varphi^{1}(x)$ is finite and the Gibbs distribution $P_{V}^{1}(\varphi(x))$ on the set $\Phi(V)$ is well defined.

Let $\varphi_{V}^{\min , 1}(x) \in \Phi(V)$ be a configuration with the minimal energy:

$$
H\left(\varphi_{V}^{\min , 1}(x) \mid \varphi^{1}(x)\right)=\min _{\varphi(x) \in \Phi(V)} H\left(\varphi(x) \mid \varphi^{1}(x)\right) .
$$

Then the configuration $\varphi_{\min }(x)$ almost coincides with the ground state of model (1) (see Lemma 1 in Section 3). Due to condition (2) the difference between energies of two minimal configurations $\varphi_{\min }$ and $\varphi_{\min }^{\prime}$ corresponding to different boundary conditions is bounded by some constant. Thus, we can define a common (for all boundary conditions) contour (a contour will be defined as a connected subconfiguration not 
coinciding with the ground state) model and by using a well-known trick [14] we come to noninteracting clusters from interacting contours.

Consider an arbitrary segment $I$ in the volume $I_{V}$, two arbitrary boundary conditions $\varphi^{1}(x)$ and $\varphi^{2}(x)$. We prove that the dependence of the expression $\mathbf{P}^{1}\left(\varphi^{\prime}(I)\right) / \mathbf{P}^{2}\left(\varphi^{\prime}(I)\right)$ on the boundary conditions $\varphi^{1}(x)$ and $\varphi^{2}(x)$ can be reduced to the sum of statistical weights of super clusters connecting the segment $I$ with the boundary and this expression is negligible at low temperatures.

Therefore, two arbitrary extreme Gibbs states are mutually continuous and hence coincide.

\section{Proof of Theorem 1}

Let $\varphi_{V}^{\min , 1}(x) \in \Phi(V)$ be a configuration with the minimal energy. The following lemma describes the structure of the configuration $\varphi_{V}^{\min , 1}(x)$.

Lemma 1. For arbitrary fixed boundary conditions $\varphi^{1}(x)$ there exist positive constants $N_{b}^{l}$ and $N_{b}^{r}$ not depending on the boundary conditions $\varphi^{1}(x)$ and $V$, such that the restriction of the configuration $\varphi_{V}^{\min , 1}(x)$ on interval $\left[-V+N_{b}^{l}, V-N_{b}^{r}\right]$ coincides with the ground state $\varphi^{g r}(x)$.

It can be easily shown that the lemma follows from condition (22). For a detailed proof of this statement see [9]. Below we give a proof of the lemma in the special translationally invariant potential case. This proof is rather amusing due to the fact that it does not employ any of the conditions (2), (3) and (22) and uses only the very natural condition that the potential tends to zero while the distance between interacting elements tends to infinity.

Proof. Obviously, for each value of $V$ there are numbers $N_{b}^{l}\left(V, \varphi^{1}\right)$ and $N_{b}^{r}\left(V, \varphi^{1}\right) \mathrm{p}$ $\left(0 \leqslant N_{b}^{l}\left(V, \varphi^{1}\right) \leqslant V, 0 \leqslant N_{b}^{r}\left(V, \varphi^{1}\right) \leqslant V\right)$ satisfying the lemma, thus, the restriction of the configuration $\varphi_{V}^{\min , 1}(x)$ to the set $\left[-V+N_{b}^{l}, V-N_{b}^{r}\right]$ coincides with the ground state $\varphi^{g r}(x)$.

Let $N_{b}^{l}\left(V, \varphi^{1}\right)$ and $N_{b}^{r}\left(V, \varphi^{1}\right)$ be minimal, that is $N_{b}^{l}\left(V, \varphi^{1}\right)+N_{b}^{r}\left(V, \varphi^{1}\right)$ is minimal.

Let $N_{b}\left(V, \varphi^{1}\right)=\max \left(N_{b}^{l}\left(V, \varphi^{1}\right), N_{b}^{r}\left(V, \varphi^{1}\right)\right)$ and

$$
N_{b}(V)=\max _{\varphi^{1}} N_{b}\left(V, \varphi^{1}\right),
$$

where the second maximum is taken over all possible boundary conditions $\varphi^{1}$.

In order to prove the lemma we show that $\max _{V} N_{b}(V)$ is bounded.

Indeed, suppose that $\max _{V} N_{b}(V)$ is not bounded. Then there exist a sequence of numbers $V(k)$, a sequence of boundary conditions $\varphi^{k}(x) ; x \in \mathbf{Z}^{1}-\left[-V_{k}, V_{k}\right]$ and corresponding sequence of configurations with minimal energy $\varphi_{V(k)}^{\min , k}(x), k=1,2, \ldots$, such that $\lim _{k \rightarrow \infty} V(k)=\infty$ and $\lim _{k \rightarrow \infty} N_{b}\left(V(k), \varphi^{k}\right)=\infty$.

Without loss of generality, we assume that $\lim _{k \rightarrow \infty} N_{b}^{l}\left(V(k), \varphi^{k}\right)=\infty$. 
Define a maximal nonnegative integer number $z=z\left(V(k), \varphi^{k}\right)$ satisfying the condition:

$$
\varphi_{V(k)}^{\min , k}\left(\left[-V(k)+N_{b}^{l}-z,-V(k)+N_{b}^{l}\right]\right) \neq \varphi^{g r}\left(\left[-V(k)+N_{b}^{l}-z,-V(k)+N_{b}^{l}\right]\right) .
$$

Due to the definitions, $z=z\left(V(k), \varphi^{k}\right)>0$, if $k$ is sufficiently large. Below we assume that $z=z\left(V(k), \varphi^{k}\right)>0$.

Now we are faced with two possible cases.

Case 1. $\lim _{k^{\prime} \rightarrow \infty} z\left(V\left(k^{\prime}\right), \varphi^{k^{\prime}}\right)+\left(V\left(k^{\prime}\right)-N_{b}^{r}\right)-\left(-V\left(k^{\prime}\right)+N_{b}^{l}\right)=\infty$ for some subsequence $k^{\prime}$ of $k$.

Case 2. $\max _{k} z\left(V(k), \varphi^{k}\right)+\left(V\left(k^{\prime}\right)-N_{b}^{r}\right)-\left(-V\left(k^{\prime}\right)+N_{b}^{l}\right)$ is bounded, where the maximum is taken over all values of $k$.

Let us define a configuration $\psi_{V\left(k^{\prime}\right)}(x)=\varphi_{V(k)}^{\min , k}(x-\bar{x})$.

In the first case we put $\bar{x}=V\left(k^{\prime}\right)-N_{b}^{l}+z / 2$. Thus, $\psi_{V\left(k^{\prime}\right)}(x)$ is a $V\left(k^{\prime}\right)-N_{b}^{l}-z / 2$ shift of $\varphi_{V\left(k^{\prime}\right)}^{\min , k^{\prime}}(x)$ to the right.

In the second case we put $\bar{x}=V\left(k^{\prime}\right)-N_{b}^{l} / 2$. Thus, $\psi_{V\left(k^{\prime}\right)}(x)$ is a $V\left(k^{\prime}\right)-N_{b}^{l} / 2$ shift of $\varphi_{V\left(k^{\prime}\right)}^{\min , k^{\prime}}(x)$ to the right.

Now note that

(1) In both cases the support of the configurations $\psi_{V\left(k^{\prime}\right)}(x)$ infinitely grows in both directions when $V\left(k^{\prime}\right)$ goes to infinity.

(2) In both cases the restriction of the configurations $\psi_{V\left(k^{\prime}\right)}(x)$ to any interval $[-L, L]$ (when $L$ is sufficiently large) does not coincide with the ground state:

$$
\psi_{V\left(k^{\prime}\right)}([-L, L]) \neq \varphi^{g r}([-L, L]) .
$$

To verify the first property we have to show that in the configuration $\varphi_{V\left(k^{\prime}\right)}^{\min , k^{\prime}}(x)$ the distances $\operatorname{dist}\left(-V\left(k^{\prime}\right),-\bar{x}\right)$ and $\operatorname{dist}\left(-\bar{x}, V\left(k^{\prime}\right)\right)$ tend to infinity in both cases.

The first property readily follows from the definitions.

In the first case, $\operatorname{dist}\left(-V\left(k^{\prime}\right),-\bar{x}\right) \geqslant N_{b}^{l} / 2$ in both cases and since $N_{b}^{l}$ tends to infinity the expression $\operatorname{dist}\left(-V\left(k^{\prime}\right),-\bar{x}\right)$ unboundedly grows. The expression $\operatorname{dist}\left(-\bar{x}, V\left(k^{\prime}\right)\right) \geqslant$ $z / 2+\left(V\left(k^{\prime}\right)-N_{b}^{r}\right)-\left(-V\left(k^{\prime}\right)+N_{b}^{l}\right)$ and obviously tends to infinity in the first case. In the second case we have to show that both distances $\operatorname{dist}\left(-\bar{x}, V\left(k^{\prime}\right)\right)$ and $\operatorname{dist}\left(-V\left(k^{\prime}\right),-\bar{x}\right)$ unboundedly grow when $N_{b}^{l}$ tends to infinity. It directly follows from the fact that $\operatorname{dist}\left(-\bar{x}, V\left(k^{\prime}\right)\right) \geqslant N_{b}^{l} / 2$ and $\operatorname{dist}\left(-V\left(k^{\prime}\right),-\bar{x}\right)=N_{b}^{l} / 2$. The first property is proved.

The second property in the first case readily follows from the definition of $z$.

In the second case assume that there is a segment $[-L, L]$ such that the restriction of $\psi(x)$ to the interval $[-L, L]$ coincides with some ground state $\varphi(x)$. Then by definition of $N_{b}^{l}$ and $N_{b}^{r}\left(N_{b}^{l}\left(V, \varphi^{k}\right)+N_{b}^{r}\left(V, \varphi^{k}\right)\right.$ is minimal) $2 L \leqslant\left(V\left(k^{\prime}\right)-N_{b}^{r}\right)-\left(-V\left(k^{\prime}\right)+N_{b}^{l}\right)$ and since $z / 2+\left(V\left(k^{\prime}\right)-N_{b}^{r}\right)-\left(-V\left(k^{\prime}\right)+N_{b}^{l}\right)$ is bounded (over the set of all $\left.k^{\prime}\right) 2 L$ is bounded and the second property is held.

We say that a sequence of configurations $\psi_{V(k)}(x)$ point-wisely converges to the configuration $\psi(x)$, if for each $x \in \mathbf{Z}^{1}$, there exists $k_{1}$, such that $\psi_{V(k)}(x)=\psi(x)$, if $k>k_{1}$. 
After this natural definition, by using a diagonal argument we can show that the sequence $\psi_{V\left(k^{\prime}\right)}(x), k^{\prime}=1,2, \ldots$ has at least one limit point, say $\psi^{\min }(x) \neq \varphi^{g r}$. Indeed, there exists a subsequence $\psi_{V\left(k^{\prime}\right)}^{0}(x)$ of $\psi_{V\left(k^{\prime}\right)}(x)$, such that $\psi_{V\left(k^{\prime}\right)}^{0}(0)$ is a constant. There exists a subsequence $\psi_{V\left(k^{\prime}\right)}^{0,1}(x)$ of $\psi_{V\left(k^{\prime}\right)}^{0}(x)$, such that $\psi_{V\left(k^{\prime}\right)}^{0,1}(1)$ is a constant. There exists a subsequence $\psi_{V\left(k^{\prime}\right)}^{0,1,-1}(x)$ of $\psi_{V\left(k^{\prime}\right)}^{0,1}(x)$, such that $\psi_{V\left(k^{\prime}\right)}^{0,1,-1}(-1)$ is a constant. By continuing this process we obtain a subsequence $\psi_{V\left(k^{\prime}\right)}^{0.1,-., n,-n, \ldots}(x)$ of $\psi_{V(k)}(x)$ which converges to some configuration $\psi^{\min }(x)$.

Now, note that $\psi^{\min }(x)$ is a ground state. In fact, suppose that $\psi(x)$ is an arbitrary perturbation of $\psi^{\min }(x)$ on some finite set $W$.

$$
\begin{aligned}
H(\psi(x))-H\left(\psi^{\min }(x)\right) \geqslant & H_{V}\left(\varphi(x) \mid \varphi^{k^{\prime}}(x)\right)-H_{V}\left(\varphi^{\min }(x) \mid \varphi^{k^{\prime}}(x)\right) \\
& -\varepsilon\left(W, V(k), \varphi^{k^{\prime}}\right),
\end{aligned}
$$

where $\varphi(x)$ is the same perturbation of $\varphi^{\min }(x)$ on the set $W-\bar{x}$, and for each fixed $W$ the term $\varepsilon\left(W, V\left(k^{\prime}\right), \varphi^{k^{\prime}}\right)$ tends to zero uniformly with respect to $\varphi^{k^{\prime}}$ while $V\left(k^{\prime}\right)$ tends to infinity.

But by construction $H_{V}\left(\varphi(x) \mid \varphi^{k^{\prime}}\right)-H_{V}\left(\varphi^{\min , k^{\prime}}(x) \mid \varphi^{k^{\prime}}\right) \geqslant 0$. Therefore, $H(\varphi(x))-$ $H\left(\varphi^{\min }(x)\right) \geqslant 0$ and $\psi^{\min }(x)$ is a ground state.

Now, note that the configuration $\psi^{\min }(x) \neq \varphi^{g r}(x)$ due to the second property. In fact, since the configuration $\psi_{V\left(k^{\prime}\right)}(x)$, which is just a shift of $\varphi_{V\left(k^{\prime}\right)}^{\min , k^{\prime}}$, the ground state $\varphi^{g r}$ cannot coincide with $\psi_{V\left(k^{\prime}\right)}(x)$ on the interval $[-L, L]$. And $\psi^{\min }(x)$ is a limit of configurations $\psi_{V\left(k^{\prime}\right)}(x)$.

This contradicts the assumption that $\max _{V} N_{b}(V)$ is not bounded. Lemma 1 is proved.

Consider the partition of $\mathbf{Z}^{1}$ into segments $I_{k}$, where $I_{k}$ is a segment with the center at $x=\frac{1}{2}+k$ and with the length 1 .

Let us consider an arbitrary configuration $\varphi(x)$. We say that a segment $I_{k}$ is not regular, if there exists a segment $I_{k}^{\prime}$, connected with $I_{k}$, such that $\varphi\left(I_{k}^{\prime}\right) \neq \varphi^{g r}\left(I_{k}^{\prime}\right)$. Two nonregular segments are called connected provided their intersection is not empty. The connected components of nonregular segments defined in such a way are called supports of contours and are denoted as $\operatorname{supp} K$.

The pair $K=(\operatorname{supp} K, \varphi(\operatorname{supp} K))$ is called a contour.

Let $\mathbf{P}^{1}$ and $\mathbf{P}^{2}$ be two Gibbs states of model (1) corresponding to the boundary conditions $\varphi^{1}(x)$ and $\varphi^{2}(x)$, respectively.

Lemma 2. Gibbs measures $\mathbf{P}^{1}$ and $\mathbf{P}^{2}$ are absolutely continuous with respect to each other.

Proof. Let $I=[a, b]$ be an arbitrary segment and $\varphi^{\prime}(I)$ be an arbitrary configuration.

In order to prove the lemma we show that there exist two positive constants $s$ and $S$ not depending on $I, \varphi^{1}(x), \varphi^{2}(x)$ and $\varphi^{\prime}(I)$, such that

$$
s \leqslant \mathbf{P}^{1}\left(\varphi^{\prime}(I)\right) / \mathbf{P}^{2}\left(\varphi^{\prime}(I)\right) \leqslant S .
$$


Let $\mathbf{P}_{\mathbf{V}}^{1}$ and $\mathbf{P}_{\mathbf{V}}^{2}$ be Gibbs measures corresponding to the boundary conditions $\varphi^{1}(x)$, and $\varphi^{2}(x), x \in \mathbf{Z}^{1}-I_{V}$, respectively, where $\mathbf{Z}^{1}-I_{V}=(-\infty,-V-1] \cup[V+1,+\infty)$.

Thus,

$$
\lim _{V \rightarrow \infty} \mathbf{P}_{V}^{1}=\mathbf{P}^{1} \quad \text { and } \quad \lim _{V \rightarrow \infty} \mathbf{P}_{\mathbf{V}}^{2}=\mathbf{P}^{2},
$$

where by convergence we mean weak convergence of probability measures.

For establishing inequality (5) we prove that for each fixed interval $I$, there exists a number $V_{0}(I)$, depending on $I$ only, such that for $V>V_{0}$

$$
s \leqslant \mathbf{P}_{\mathbf{V}}^{1}\left(\varphi^{\prime}(I)\right) / \mathbf{P}_{\mathbf{V}}^{2}\left(\varphi^{\prime}(I)\right) \leqslant S .
$$

Let $H\left(\varphi(x) \mid \varphi^{1}(x), \varphi^{\min , 1}(x)\right)$ denote the relative energy of a configuration $\varphi(x)$ (with respect to $\left.\varphi^{\min , 1}(x)\right)$ :

$$
H\left(\varphi(x) \mid \varphi^{1}(x), \varphi^{\min , 1}(x)\right)=H\left(\varphi(x) \mid \varphi^{1}(x)\right)-H\left(\varphi^{\min , 1}(x) \mid \varphi^{1}(x)\right) .
$$

Consider

$$
\begin{aligned}
\mathbf{P}_{\mathbf{V}}^{1}\left(\varphi^{\prime}(I)\right) & =\frac{\sum_{\varphi\left(I_{V}\right): \varphi(I)=\varphi^{\prime}(I)} \exp \left(-\beta H\left(\varphi\left(I_{V}\right) \mid \varphi^{1}(x), \varphi^{\min , 1}(x)\right) Y\left(\varphi^{\prime}(I), V, \varphi^{1}(x)\right)\right)}{\sum_{\varphi\left(I_{V}\right)} \exp \left(-\beta H\left(\varphi\left(I_{V}\right) \mid \varphi^{1}(x), \varphi^{\min , 1}(x)\right) Y\left(\varphi(I), V, \varphi^{1}(x)\right)\right)} \\
& =\frac{\Xi\left(I_{V}-I \mid \varphi^{1}(x), \varphi^{\prime}(I), \varphi^{\min , 1}(x)\right) Y\left(\varphi^{\prime}(I), V, \varphi^{1}(x)\right)}{\sum_{\varphi^{\prime \prime}(I)} \Xi\left(I_{V}-I \mid \varphi^{1}(x), \varphi^{\prime \prime}(I), \varphi^{\min , 1}(x)\right) Y\left(\varphi^{\prime \prime}(I), V, \varphi^{1}(x)\right)} \\
& =\frac{\Xi^{1, \prime} Y\left(\varphi^{\prime}(I), V, \varphi^{1}(x)\right)}{\sum_{\varphi^{\prime \prime}(I)} \Xi^{1,^{\prime \prime}} Y\left(\varphi^{\prime \prime}(I), V, \varphi^{1}(x)\right)},
\end{aligned}
$$

where $\Xi^{1, \prime}=\Xi\left(I_{V}-I \mid \varphi^{1}(x), \varphi^{\prime}(I), \varphi^{\min , 1}(x)\right)$ denotes the partition function corresponding to the boundary conditions $\varphi^{1}(x), x \in \mathbf{Z}^{1}-I_{V}, \varphi^{\prime}(I), x \in I$ and

$$
\begin{aligned}
& Y\left(\varphi(I), V, \varphi^{i}(x)\right) \\
& \quad=\sum_{A \subset \mathbf{Z}^{1}: A \cap I \neq \emptyset ; A \cap \mathbf{Z}^{1}-I_{V} \neq \emptyset} \exp \left(-\beta\left(U(\varphi(A))-U\left(\varphi^{\min , i}(A)\right)\right)\right), \quad i=1,2,
\end{aligned}
$$

where $\varphi(x)$ in sum (6) is equal to $\varphi^{\prime}(x)$ for $x \in I$ and it is equal to $\varphi^{i}$ for $x \in \mathbf{Z}^{1}-I_{V}$. Expression (7) gives the "direct" interaction of $\varphi(I)$ with the boundary condition $\varphi^{i}$. We can express $\mathbf{P}_{\mathbf{V}}^{2}\left(\varphi^{\prime}(I)\right)$ in just the same way.

In order to prove inequality (6) it is enough to establish inequalities (8) and (9):

$$
1<Y\left(\varphi(I), V, \varphi^{i}(x)\right)<2 ; \quad i=1,2
$$

and

$$
1 / S \leqslant\left(\frac{\Xi^{1, \prime \prime}}{\Xi^{1, \prime}}\right) /\left(\frac{\Xi^{2, \prime \prime}}{\Xi^{2, \prime}}\right) \leqslant 1 / s
$$

for arbitrary $\varphi^{\prime \prime}(I)$. 
Indeed, if inequalities (8) and (9) hold, then

$$
1 /(1 / s) \leqslant \mathbf{P}_{\mathbf{V}}^{1}\left(\varphi^{\prime}(I)\right) / \mathbf{P}_{\mathbf{V}}^{2}\left(\varphi^{\prime}(I)\right) \leqslant 1 /(1 / S),
$$

since the quotient of $\left(\sum_{i=1}^{n} a_{i}\right) /\left(\sum_{i=1}^{n} b_{i}\right)$ lies between $\min \left(a_{i} / b_{i}\right)$ and $\max \left(a_{i} / b_{i}\right)$.

Now, we start to prove inequalities (8) and (9).

Inequality (8) is a direct consequence of the natural condition on the decreasing of the potential: for each fixed $I$ there exists $V_{0}$, such that if $V>V_{0}$, then $1<Y(\varphi(I), V$, $\left.\varphi^{i}(x)\right)<2 ; i=1,2$.

So, in order to complete the proof of Lemma 2 we have to establish the following inequality (which is just the transformed inequality (9)):

$$
1 / S \leqslant \frac{\Xi^{1, \prime \prime} \Xi^{2, \prime}}{\Xi^{2, \prime \prime} \Xi^{1, \prime}} \leqslant 1 / s .
$$

Define a super partition function

$$
\begin{aligned}
\left(\Xi^{1, \prime \prime} \Xi^{2, \prime}\right)= & \sum \exp \left(-\beta H\left(\varphi^{3}\left(I_{V}\right) \mid \varphi^{1}(x), \varphi^{\prime \prime}(x) \varphi^{\min }(x)\right)\right) \\
& \times \exp \left(-\beta H\left(\varphi^{4}\left(I_{V}\right) \mid \varphi^{2}(x), \varphi^{\prime}(x) \varphi^{\min }(x)\right)\right),
\end{aligned}
$$

where the summation in $\sum_{p}$ is taken over all pairs of configurations $\varphi^{3}\left(I_{V}\right)$ and $\varphi^{4}\left(I_{V}\right)$, such that $\varphi^{3}(I)=\varphi^{\prime \prime}(x) ; \varphi^{4}(I)=\varphi^{\prime}(x)$.

Now, we show that for each fixed interval $I$, there exists a number $V_{0}(I)$, which depends on $I$ only, such that if $V>V_{0}(I)$

$$
s \leqslant\left(\Xi^{1, \prime} \Xi^{2, \prime \prime}\right) /\left(\Xi^{1, \prime \prime} \Xi^{2, \prime}\right) \leqslant S
$$

for two positive constants $s$ and $S$ not depending on $I, \varphi^{1}(x), \varphi^{2}(x), \varphi^{\prime}(x)$ and $\varphi^{\prime \prime}(x)$.

Now, we try to represent the super partition functions $\left(\Xi^{1, \prime} \Xi^{2, \prime \prime}\right)$ and $\left(\Xi^{1, \prime \prime} \Xi^{2, \prime}\right)$ in a more convenient form. Roughly speaking, by using a well-known technique we are going to pass to noninteracting clusters from interacting contours [14].

Let the boundary conditions $\bar{\varphi}(x)=[\varphi(x), x \in(-\infty,-V-1] \cup[V+1, \infty)]$ be fixed. As above the set of all configurations $\varphi(x) ; x \in[-V, V]$ we denote by $\Phi(V)$.

It is obvious that for each contour $K$, such that $\operatorname{supp} K \in\left[-V+N_{b}, V-N_{b}\right]$, there exists a configuration $\psi_{K}([-V, V])$ such that the only contour of the configuration $\psi_{K}([-V, V])$ is $K$.

The weight of contour $K$ will be calculated by the following formula:

$$
\gamma(K)=H\left(\psi_{K}(x)\right)-H\left(\varphi^{1}(x)\right) .
$$

Consider the Gibbs distribution $\mathbf{P}^{1}$ on $\Phi(V)$ corresponding to the boundary conditions $\varphi^{1}(x)=\left[\varphi^{1}(x), x \in(-\infty,-V-1] \cup[V+1, \infty)\right]$ :

$$
\mathbf{P}^{1}\left(\varphi^{\prime}(x)\right)=\frac{\exp \left(-\beta\left(H\left(\varphi^{\prime}(x) \mid \varphi^{1}(x), \varphi^{\min , 1}(x)\right)\right)\right)}{\sum_{\varphi(x) \in \Phi(V)} \exp \left(-\beta\left(H\left(\varphi(x) \mid \varphi^{1}(x), \varphi^{\min , 1}(x)\right)\right)\right)} .
$$


Let $\varphi(x) \in \Phi(V)$ be an arbitrary configuration, the boundary of the $\varphi(x)$ includes a finite number of contours $K_{i} ; i=1, \ldots, n$. The set of all contours of the boundary conditions $\varphi^{1}(x)$ will be denoted by $K_{0}$.

The statistical weight of a contour is

$$
w\left(K_{i}\right)=\exp \left(-\beta \gamma\left(K_{i}\right)\right) .
$$

The following equation is a direct consequence of formulas (12) and (14)

$$
\exp \left(-\beta H\left(\varphi(x) \mid \varphi^{1}(x), \varphi^{\min , 1}(x)\right)\right)=\prod_{i=1}^{n} w\left(K_{i}\right) \exp \left(-\beta G\left(K_{0}, K_{1}, \ldots, K_{n}\right)\right),
$$

where the multiplier $G\left(K_{0}, K_{1}, \ldots, K_{n}\right)$ corresponds to the interaction between contours and with the boundary conditions $\varphi^{1}(x)$ :

$$
G\left(K_{0}, K_{1}, \ldots, K_{n}\right)=\sum_{k=2}^{n} \sum_{i_{1}, \ldots, i_{k}} G\left(K_{i_{1}}, \ldots, K_{i_{k}}\right)=\sum_{k=2}^{n} \sum_{(B) \in \operatorname{Int}\left(K_{i_{1}}, \ldots, K_{i_{k}}\right)} f(B),
$$

where at each fixed $k$ the summation is taken over all possible collections $i_{1}, \ldots, i_{k}$, $i_{j}=0, \ldots, n, i_{l}<i_{m}$, if $l<m$.

The interaction between some point $x$ from the support of $K_{1}$ and some point $y$ from the support of $K_{2}$ arises due to the fact that the weight of the contour $K_{1}$ was calculated under assumption that the configuration outside $\operatorname{supp}\left(K_{1}\right)$ coincides with the ground state.

We do not need the explicit expressions of $f(B)$ and $\left(\operatorname{int}\left(K_{i}, K_{j}\right)\right)$, they are very huge and we do not write them down. For the pair potential case see [9].

For simplicity, $K_{i}, i=1, \ldots, n$ will be denoted by $K_{i}, i \in \mathbf{I}$. Thus, formula (15) has the form

$$
\exp \left(-\beta H\left(\varphi(x) \mid \varphi^{1}(x), \varphi^{\min , 1}(x)\right)\right)=\prod_{i \in \mathbf{I}} w\left(K_{i}\right) \exp \left(-\beta G\left(K_{0}, K_{1}, \ldots, K_{n}\right)\right) .
$$

The set of all interaction elements $B$ in the double sum (16) will be denoted by $I G$ (for the pair potential $B$ will be a pair of points $(x, y)$ ). Write Eq. (17) as follows:

$$
\exp \left(-\beta H\left(\varphi(x) \mid \varphi^{1}(x), \varphi^{\min , 1}(x)\right)\right)=\prod_{i \in \mathbf{I}} w\left(K_{i}\right) \prod_{B \in I G}(1+\exp (-\beta f(B)-1)) .
$$

From Eq. (18) we get

$$
\exp \left(-\beta H\left(\varphi(x) \mid \varphi^{1}(x), \varphi^{\min , 1}(x)\right)\right)=\sum_{I G^{\prime} \subset I G} \prod_{i \in \mathbf{I}} w\left(K_{i}\right) \prod_{B \in I G^{\prime} ; f(B) \neq 0} g(B),
$$

where the summation is taken over all subsets $I G^{\prime}$ (including the empty set) of the set $I G$, and $g(B)=\exp (-\beta f(B))-1$.

Consider an arbitrary term of sum (19), which corresponds to the subset $I G^{\prime} \subset I G$. Let the interaction element $B \in I G^{\prime}$. Consider the set $\mathbf{K}$ of all contours such that for each 
contour $K \subset \mathbf{K}$, the set $\operatorname{supp} K \cap B$ contains one point. We call any two contours from $\mathbf{K}$ connected.The set of contours $K^{\prime}$ is called $I G^{\prime}$ connected if for any two contours $K_{p}$ and $K_{q}$ there exists a collection $\left(K_{1}=K_{p}, K_{2}, \ldots, K_{n}=K_{q}\right)$ such that any two contours $K_{i}$ and $K_{i+1}, i=1, \ldots, n-1$, are connected by some interaction element $B \in I G^{\prime}$.

The pair $D=\left[\left(K_{i}, i=1, \ldots, s\right) ; I G^{\prime}\right]$, where $I G^{\prime}$ is some set of interaction elements, is called a cluster provided there exists a configuration $\varphi(x)$ containing all $K_{i} ; i=1, \ldots, s$; $I G^{\prime} \subset I G$; and the set $\left(K_{i}, i=1, \ldots, s\right)$ is $I G^{\prime}$ connected. The statistical weight of a cluster $D$ is defined by the formula

$$
w(D)=\prod_{i=1}^{s} w\left(K_{i}\right) \prod_{(x, y) \in I G^{\prime}} g(B),
$$

Note that $w(D)$ is not necessarily positive.

Two clusters $D_{1}$ and $D_{2}$ are called compatible provided any two contours $K_{1}$ and $K_{2}$ belonging to $D_{1}$ and $D_{2}$, respectively, are compatible. A set of clusters is called compatible provided any two clusters of it are compatible.

If $D=\left[\left(K_{i}, i=1, \ldots, s\right) ; I G^{\prime}\right]$, then we say that $K_{i} \in D ; i=1, \ldots, s$.

The following lemma is a direct consequence of the definitions.

Lemma 3. Let boundary conditions $\varphi^{1}(x)=\left[\varphi^{1}(x), x \in(-\infty,-V-1] \cup[V+1, \infty)\right]$ be fixed.

If $\left[D_{1}, \ldots, D_{m}\right]$ is a compatible set of clusters and $\bigcup_{i=1}^{m} \operatorname{supp} D_{i} \subset[-V, V]$, then there exists a configuration $\varphi(x)$ which contains this set of clusters. For each configuration $\varphi(x)$ we have

$$
\exp \left(-\beta H\left(\varphi(x) \mid \varphi^{1}(x), \varphi^{\min , 1}(x)\right)\right)=\sum_{I G^{\prime} \subset I G} \prod w\left(D_{i}\right),
$$

where the clusters $D_{i}$ are completely determined by the set $I G^{\prime}$. The partition function is

$$
\Xi\left(\varphi^{1}(x)\right)=\sum w\left(D_{1}\right) \ldots w\left(D_{m}\right)
$$

where the summation is taken over all non-ordered compatible collections of clusters.

Lemma 3 shows that we come to noninteracting clusters from interacting contours.

The following generalization of the definition of compatibility allows us to represent $\left(\Xi^{1, \prime \prime} \Xi^{2, \prime}\right)$ as a single partition function.

A set of clusters is called super compatible provided any of its two parts coming from two Hamiltonians is compatible. In other words, in super compatibility an intersection of supports of two clusters is allowed.

Lemma 4. Let boundary conditions $\varphi^{1}(x)=\left[\varphi^{1}(x), x \in(-\infty,-V-1] \cup[V+1, \infty)\right]$ and $\varphi^{2}(x)=\left[\varphi^{2}(x), x \in(-\infty,-V-1] \cup[V+1, \infty)\right]$ be fixed. 
If $\left[D_{1}, \ldots, D_{m}\right]$ is a super compatible set of clusters and $\bigcup_{i=1}^{m} \operatorname{supp} D_{i} \subset[-V, V]$, then there exist two configurations $\varphi^{3}(x)$ and $\varphi^{4}(x)$ which contain this set of clusters. For each pair of configurations $\varphi^{3}(x)$ and $\varphi^{4}(x)$ we have

$$
\begin{aligned}
& \exp \left(-\beta H\left(\varphi^{3}(x) \mid \varphi^{1}(x), \varphi^{\min , 1}(x)\right)\right) \exp \left(-\beta H\left(\varphi^{4}(x) \mid \varphi^{2}(x), \varphi^{\min , 2}(x)\right)\right) \\
& =\sum_{I G^{\prime} \subset I G, I G^{\prime \prime} \subset I G} \prod w\left(D_{i}\right),
\end{aligned}
$$

where the clusters $D_{i}$ are completely determined by the sets $I G^{\prime}$ and $I G^{\prime \prime}$.

The super partition function is

$$
\Xi^{1, \prime \prime 2, \prime}=\left(\Xi^{1, \prime \prime} \Xi^{2, \prime}\right)=\sum w\left(D_{1}\right) \ldots w\left(D_{m}\right),
$$

where the summation is taken over all nonordered super compatible collections of clusters.

Lemma 4 is an analogue of Lemma 3.

Let $w\left(D_{1}\right) \ldots w\left(D_{m}\right)$ be a term of the super partition function $\Xi^{1, \prime \prime 2, \prime}$. The connected components of the collection $\left[\operatorname{supp}\left(D_{1}\right), \ldots, \operatorname{supp}\left(D_{m}\right)\right]$ are the supports of the super clusters. A super cluster $S D$ is a pair $(\operatorname{supp}(S D), \varphi(\operatorname{supp}(S D)))$. Below, instead of the expression "super compatible collection of clusters" we use the expression "compatible collection of super clusters".

A cluster (a super cluster) $D=\left[\left(K_{i}, i=1, \ldots, r\right) ; I G^{\prime}\right]\left(S D=\left[\left(K_{i}, i=1, \ldots, r\right) ; I G^{\prime}\right]\right)$ is said to be long if the intersection of the set $\left.\left(\bigcup_{i=1}^{m} \operatorname{supp} K_{i}\right)\right) \cup I G^{\prime}$ with both $I$ and $\mathbf{Z}^{1}-I_{V}=(-\infty,-V-1] \cup[V+1, \infty)$ is nonempty. In other words, a long cluster (super cluster) connects the boundary with the segment $I$.

A set of super clusters is called compatible provided the set of all clusters belonging to these super clusters are super compatible.

The following important lemma shows that in our estimates long super clusters are negligible.

Lemma 5. For each fixed interval $I$, there exists a number $V_{0}(I)$, which depends on $I$ only, such that if $V>V_{0}(I)$

$$
\frac{1}{2} \Xi^{1, \prime, 2, \prime \prime}<\Xi^{1, \prime, 2, \prime \prime,(n . l .)}=\sum w\left(S D_{1}\right) \cdots w\left(S D_{m}\right),
$$

where the summation is taken over all nonlong, nonordered compatible collections of super clusters $\left[S D_{1}, \ldots, S D_{m}\right], \bigcup_{i=1}^{m} \operatorname{supp}\left(S D_{i}\right) \subset I_{N}-I$ corresponding to the boundary conditions $\varphi^{1}(x), \varphi^{2}(x), x \in \mathbf{Z}^{1}-I_{V} ; \varphi^{\prime}(x)$ and $\varphi^{\prime \prime}(x), x \in I$.

Consider a collection of contours $K_{0}, K_{1}, \ldots, K_{n}$. The value of the interaction of the contour $K_{0}$ with the contours $K_{1}, \ldots, K_{n}$ we denote via $G\left(K_{0} \mid K_{1}, \ldots, K_{n}\right)$ :

$$
G\left(K_{0} \mid K_{1}, \ldots, K_{n}\right)=\prod_{B \in I G(0 \mid 1, \ldots, n)}(1+\exp (-\beta f(B)-1))
$$


where $I G(0 \mid 1, \ldots, n)$ is the set of all interaction elements intersecting the support of the contour $K_{0}$.

On the potential $U(B)$ we impose the following natural condition:

$$
\begin{aligned}
G\left(K_{0} \mid K_{1}, \ldots, K_{n}\right) & =\prod_{B \in I G(0 \mid 1, \ldots, n)} \mid(1+\exp (-\beta f(B)-1)) \\
& \leqslant h_{1}\left(\left|\operatorname{supp}\left(K_{0}\right)\right|\right) h_{2}(\operatorname{dist}(0 \mid 1, \ldots, n)),
\end{aligned}
$$

where $\operatorname{dist}(0 \mid 1, \ldots, n)$ is the distance between the support of $K_{0}$ and the union of the supports of contours $K_{1}, \ldots, K_{n}$, and the functions $h_{i}(x)$ satisfy the following conditions:

$$
\lim _{x \rightarrow \infty} h_{1}(x) / x=0 \quad \lim _{x \rightarrow \infty} h_{2}(x)=0 .
$$

In other words, the interaction of $K_{1}, \ldots, K_{n}$ on $K_{0}$ tends to zero when the distance between them increases, and the value of the interaction increases with a rate less than the length of the support of $K_{0}$.

These conditions are very natural and in particular are held in all models with pair potential $U(x) \sim 1 / x^{1+\alpha}$, as $x \rightarrow \infty, 0<\alpha$. In the pair potential case (see [9])

$$
G\left(K_{0} \mid K_{1}, \ldots, K_{n} \leqslant \operatorname{const}(\operatorname{dist}(0 \mid 1, \ldots, n))^{-\alpha}\left(\left|\operatorname{supp}\left(K_{0}\right)\right|\right)^{1-\alpha} .\right.
$$

The following lemma is an analogue of Lemma 5 for clusters (not super clusters).

Lemma 6. For each fixed interval $I$, there exists a number $V_{0}(I)$, which depends on $I$ only, such that if $V>V_{0}(I)$

$$
\frac{1}{2} \Xi^{1, \prime}<\Xi^{1, l,(n . l .)}=\sum w\left(D_{1}\right) \ldots w\left(D_{m}\right),
$$

where the summation is taken over all nonlong, nonordered compatible collections of clusters $\left[D_{1}, \ldots, D_{m}\right], \bigcup_{i=1}^{m} \operatorname{supp} D_{i} \subset I_{N}-I$ corresponding to the boundary conditions $\varphi^{1}(x), x \in \mathbf{Z}^{1}-I_{V} ; \varphi^{\prime}(x), x \in I$.

Proof.

$$
\Xi^{1, \prime}=\Xi^{1, l,(n . l .)}+\left(\Xi^{1, \prime}-\Xi^{1, l,(n . l .)}\right)=\Xi^{1,,(n . l .)}+\Xi^{1,,(l .)},
$$

where the summation in $\Xi^{1,,(l .)}$ is taken over all nonordered compatible collections of clusters $\left[D_{1}, \ldots, D_{m}\right]$ containing at least one long cluster, $\bigcup_{i=1}^{m} \operatorname{supp} D_{i} \subset I_{N}-I$ corresponding to the boundary conditions $\varphi^{1}(x), x \in \mathbf{Z}^{1}-I_{V} ; \varphi^{\prime}(x), x \in I$.

By dividing both sides of the last equality by $\Xi^{1, \prime}$, we get

$$
1=\Xi^{1,,(n . l .)} / \Xi^{1, \prime}+\Xi^{1, \prime,(l .)} / \Xi^{1, \prime} .
$$

Now, we are going to show that the second term (which is not necessarily positive) is negligible, that is the absolute value of it is less than $\frac{1}{2}$ (actually we can show that the absolute value of the second term is less than any fixed positive number at sufficiently large values of $V$ ). 
The term $\Xi^{1,,(l .)} / \Xi^{1, \prime}$ can be interpreted as a "probability" $P$ (Long) of the event that there exists at least one long cluster.

We show that the absolute value of this "probability" is less than $\frac{1}{2}$ by the following method. We estimate the density of long clusters: the probability that a given segment belongs to the support of some long cluster. Since some statistical weights of clusters are positive and some negative, we estimate the absolute values of these "probabilities". We show that for a fixed segment the "probability" that this segment belongs to the support of some long cluster with positive "probability" minus the "probability" that this segment belongs to the support of some long cluster with negative "probability" is less than one. Since the density is less than one, by the Law of Large Numbers a "typical" long cluster has not very long support, and therefore has long bonds. When $V$ tends to infinity, the total length of bonds tends to infinity, and the impact of these bonds tends to zero.

Let us replace each term in $\Xi^{1, \prime,(l .)}$ with its absolute value. That is, each factor $w\left(D_{i}\right)$ we replace with $\left|w\left(D_{i}\right)\right|$.

$$
\left|\Xi^{1, l,(l .)} / \Xi^{1, \prime}\right| \leqslant \sum\left|w\left(D_{1}\right)\right| \cdots\left|w\left(D_{m}\right)\right| / \Xi^{1, \prime} .
$$

Now, the expression $\sum\left|w\left(D_{1}\right)\right| \cdots\left|w\left(D_{m}\right)\right| / \Xi^{1, \prime}$ which is the sum of the absolute values of "probabilities" of configurations containing at least one long cluster can be interpreted as an "absolute probability" $P^{\text {abs }}$ (Long) of the event that there is at least one long cluster (actually, this expression exceeds the absolute value of the formal expression for the probability of the event that there is at least one long cluster).

Now, our aim is to estimate the "absolute probability" $P^{\text {abs }}$ of the event that a given segment belongs to the support of the cluster. In other words, we are going to estimate the statistical weights of clusters after replacing values of all negative bonds with their absolute values. Of course, after this replacement the statistical weights of clusters become greater, but it turns out that not essentially.

Let $\varphi(x), x \in I_{V}-I$ be an arbitrary configuration which contains contours $K_{1}, \ldots, K_{l}$, $\mathbf{K}=\bigcup_{1}^{l} \operatorname{supp}^{1} K_{i}, \mathbf{K}^{1}=\mathbf{K} \cap[-V,-(|I| / 2)]$ and $\mathbf{K}^{2}=\mathbf{K} \cap[|I| / 2, V]$.

Put $C^{1}(\varphi(x))=\left|\mathbf{K}^{1}\right|$ and $C^{2}(\varphi(x))=\left|\mathbf{K}^{2}\right|$

$$
\begin{aligned}
\mid P(\text { Long }) \mid & =\Xi^{1, \prime,(l .)} / \Xi^{1, \prime} \leqslant P^{\mathrm{abs}}(\text { Long })=\sum\left|w\left(D_{1}\right)\right| \cdots\left|w\left(D_{m}\right)\right| / \Xi^{1, \prime} \\
& =\sum^{p, 1}\left|w\left(D_{1}\right)\right| \cdots\left|w\left(D_{m}\right)\right| / \Xi^{1, \prime}+\sum^{p, 2}\left|w\left(D_{1}\right)\right| \cdots\left|w\left(D_{m}\right)\right| / \Xi^{1, \prime} \\
& =P^{\mathrm{abs}}(\text { Long },>p)+P^{\mathrm{abs}}(\text { Long }, \leqslant p)
\end{aligned}
$$

where last two summations are taken over all nonordered compatible collections of clusters $\left[D_{1}, \ldots, D_{m}\right]$ containing at least one long cluster, $\bigcup_{i=1}^{m} \operatorname{supp} D_{i} \subset I_{N}-I$ corresponding to the boundary conditions $\varphi^{1}(x), x \in \mathbf{Z}^{1}-I_{V} ; \varphi^{\prime}(x), x \in I$, the summation in $\sum^{p, 1}$ is taken over all configurations $\varphi\left(I_{V}\right): \varphi(I)=\varphi^{\prime}(I) ; 2 C^{1}(\varphi(x)) /\left(\left|I_{V}\right|-|I|\right)>p$; $2 C^{2}(\varphi(x)) /\left(\left|I_{V}\right|-|I|\right)>p$, the summation in $\sum^{p, 2}$ is taken over all configurations 
$\varphi\left(I_{V}\right): \varphi(I)=\varphi^{\prime}(I) ; 2 C^{1}(\varphi(x)) /\left(\left|I_{V}\right|-|I|\right) \leqslant p ; 2 C^{2}(\varphi(x)) /\left(\left|I_{V}\right|-|I|\right) \leqslant p$. It means that the density of contours in each configuration from $\sum^{p, 1}\left(\sum^{p, 2}\right)$ in both segments $[-V,-(|I| / 2)]$ and $[|I| / 2, V]$ is greater than $p$ (is not greater than $p$ ).

We fixed the value of $p$ as $1-q / 2 l$, where the values of $q$ and $l$ will be defined in the proof of Lemma 8 .

It turns out that the long clusters are negligible:

Lemma 7. For each fixed interval $I$ there exists a value of $V_{0}$, such that if $V>V_{0}$

$$
P^{\mathrm{abs}}(\text { Long })=P^{\mathrm{abs}}(\text { Long, }>p)+P^{\mathrm{abs}}(\text { Long, } \leqslant p)<\frac{1}{2} .
$$

Lemma 7 is a consequence of the following two lemmas.

Lemma 8. For each fixed interval $I$ there exists a value of $V_{0}$, such that if $V>V_{0}$

$$
P^{\text {abs }}(\text { Long, }>p)<\frac{1}{4} \text {. }
$$

Lemma 9. For each fixed interval $I$ there exists a value of $V_{0}$, such that if $V>V_{0}$

$$
P^{\text {abs }}(\text { Long, } \leqslant p)<\frac{1}{4} \text {. }
$$

Proof of Lemma 8. Consider the partition of $\mathbf{Z}^{1}$ into segments $T_{k}=T_{k}(l)$, where $T_{k}(l)$ is the segment with the center at $x=(l / 2)+k l$ and with the length $l\left(T_{k}\right.$ consists of $l$ segments $I_{k}$ ). The value of $l$ will be defined later. Let us consider an arbitrary configuration $\varphi(x)$. We say that a segment $I_{k}$ is regular, if $\varphi\left(I_{k-1} \cup I_{k} \cup I_{k+1}\right)=\varphi^{g r}\left(I_{k-1} \cup I_{k} \cup\right.$ $\left.I_{k+1}\right)$. We say that a segment $T_{k}$ is super-regular, if $T_{k}$ contains at least one regular segment.

Let $\mathbf{P}_{\mathbf{V}}$ be a Gibbs measure corresponding to the boundary conditions $\varphi^{1}(x), x \in \mathbf{Z}^{1}$, $\varphi^{\prime}(I), x \in I$.

Let the segment $I_{V}-I$ consist of $n$ segments $T_{k} ; k=1, \ldots, n$.

We define a sample space $\Omega$ consisting of $2^{n}$ elementary events $A^{j}=[\sigma(1), \ldots, \sigma(n)]$, where $\sigma(k), k=1, \ldots, n$ takes two values: $\sigma(k)=0$ corresponds to the case when the segment $T_{k}$ is super-regular and $\sigma(k)=1$ corresponds to the case when the segment $T_{k}$ is not super-regular. On the sample space $\Omega$ we define two different probability spaces $\left(\Omega, \mathbf{P}_{1}\right)$ and $\left(\Omega, \mathbf{P}_{2}\right)$ by the following formulas:

$$
\mathbf{P}_{1}\left(A^{j}\right)=\mathbf{P}_{1}[\sigma(1), \ldots, \sigma(n)]=\mathbf{P}_{\mathbf{V}}[\sigma(1), \ldots, \sigma(n)],
$$

where $\mathbf{P}_{\mathbf{V}}$ is the Gibbs distribution $\mathbf{P}_{\mathbf{V}}$, corresponding to the boundary conditions $\varphi^{1}(x), x \in \mathbf{Z}^{1}, \varphi^{\prime}(I), x \in I$ and

$$
\mathbf{P}_{2}\left(A^{j}\right)=\mathbf{P}_{2}[\sigma(1), \ldots, \sigma(n)]=q^{n-s}(1-q)^{s},
$$

where $s$ denotes the total number of 1 entries of the vector $A^{j}=[\sigma(1), \ldots, \sigma(n)]$. 
We define a random vector $(\eta(1), \eta(2), \ldots, \eta(n))$ on the probability space $\left(\Omega, \mathbf{P}_{1}\right)$ and, respectively, a random vector $(\xi(1), \xi(2), \ldots, \xi(n))$ on the probability space $\left(\Omega, \mathbf{P}_{2}\right)$ by the formulas:

$$
\eta(k)\left(A^{j}\right)=\sigma_{k} \quad \text { and } \quad \xi(k)\left(A^{j}\right)=\sigma_{k}
$$

The random variables $\eta(k)$ and $\xi(k)$ are defined on the same sample space but on different probability spaces.

Due to the definitions, the random variables $\eta(k)$ are dependent, and the random variables $\xi(k)$ are independent and identically distributed.

Consider the two sums $\sum_{k=1}^{n} \eta(k)$ and $\sum_{k=1}^{n} \xi(k)$.

Suppose that

$$
\mathbf{P}\left(\eta(m)=1 \mid \text { any conditions outside } T_{m}\right) \leqslant 1-q .
$$

Note that $\mathbf{P}\left(\eta(m)=1 \mid\right.$ any conditions outside $\left.T_{m}\right) \leqslant 1-q=\mathbf{P}(\xi(m)=1$ and therefore the following assertion must hold.

\section{Proposition.}

$$
\mathbf{P}\left(\sum_{k \in K} \eta(k) \geqslant l\right) \leqslant \mathbf{P}\left(\sum_{k \in K} \xi(k) \geqslant l\right)
$$

for all natural values of $l$.

Proof. Let us define a new pseudo-probability function $\mathbf{P}^{\text {mixed, } K^{\prime \prime}}$ on the sample space $\Omega$ as

$$
\begin{aligned}
& \mathbf{P}^{\text {mixed }, K^{\prime \prime}}\left(A^{j}=[\sigma(1), \ldots, \sigma(n)]\right) \\
& =\mathbf{P}^{\text {mixed }, K^{\prime \prime}}\left(\eta\left(k^{\prime}\right)=\sigma\left(k^{\prime}\right), k^{\prime} \in K^{\prime} ; \xi\left(k^{\prime \prime}\right)=\sigma\left(k^{\prime \prime}\right), k^{\prime \prime} \in K^{\prime \prime}\right) \\
& =\mathbf{P}\left(\eta\left(k^{\prime}\right)=\sigma\left(k^{\prime}\right), k^{\prime} \in K^{\prime}\right) \mathbf{P}\left(\xi\left(k^{\prime \prime}\right)=\sigma\left(k^{\prime \prime}\right), k^{\prime \prime} \in K^{\prime \prime}\right) \\
& =\mathbf{P}\left(\eta\left(k^{\prime}\right)=\sigma\left(k^{\prime}\right), k^{\prime} \in K^{\prime}\right)(1-q)^{\left|K^{\prime \prime}\right|},
\end{aligned}
$$

where $K^{\prime \prime}$ is an arbitrary subset of $[1, \ldots, n], K^{\prime}=[1, \ldots, n]-K^{\prime \prime}$, and $\left|K^{\prime \prime}\right|$ is a number of elements of $K^{\prime \prime}$.

Roughly speaking, we get $\mathbf{P}^{\text {mixed, } K^{\prime \prime}}$ by "replacing" random variables $\eta\left(k^{\prime \prime}\right)$ with random variables $\xi\left(k^{\prime \prime}\right)$.

Now, we prove the following inequality:

$$
\mathbf{P}\left(\sum_{k=1}^{n} \eta(k) \geqslant l\right) \leqslant \mathbf{P}^{\text {mixed, } K^{\prime \prime}}(A(l))
$$

for any $K^{\prime \prime} \subset[1, \ldots, n]$, where the compound event $A(l)$ is the union of all elementary events $A^{j}=[\sigma(1), \ldots, \sigma(n)]$, such that for each $\left.A^{j}: \sum_{i=1}^{n} \sigma(i) \geqslant l\right)$. 
Consider an event $\sum_{k=1}^{n} \eta(k) \geqslant l$. This compound event can be represented as the union of elementary events $A^{j}=[\sigma(1), \ldots, \sigma(n)]$, such that for each elementary event the $\sum_{i=1}^{n} \sigma(i) \geqslant l$. Thus, the inequality (27) is equivalent to the following inequality:

$$
\sum \mathbf{P}(\eta(1)=\sigma(1), \ldots, \eta(n)=\sigma(n)) \leqslant \sum \mathbf{P}^{\text {mixed }, K^{\prime \prime}}\left(A^{j}\right),
$$

where both summations are taken over all possible events $A^{j}=[\sigma(1), \ldots, \sigma(n)]$, such that $\sum_{i=1}^{n} \sigma(i) \geqslant l$.

Suppose that $K^{\prime \prime}=s$ in Eq. (27). It means that we are going to "replace" a random variable $\eta(s)$ with random variable $\xi(s)$.

Summations in Eq. (28) are taken over some class of elementary events $A^{j}$. For each elementary event $A^{j}$ we have two possibilities, namely, $\sigma(s)=1$ and $\sigma(s)=0$.

Consider $A^{j}$, such that $\sigma(s)=1$ and two terms from Eq. (27) corresponding to $A^{j}$, namely a term from left hand side and a term from right hand side of (27). For this elementary event $A^{j}=[\sigma(1), \ldots, \sigma(n)]$ we have

$$
\begin{aligned}
\mathbf{P} & \eta(1)=\sigma(1), \ldots, \eta(s)=\sigma(s)=1, \ldots, \eta(n)=\sigma(n)) \\
& =\mathbf{P}(\eta(1)=\sigma(1), \ldots, \eta(s-1)=\sigma(s-1), \eta(s+1) \\
& =\sigma(s+1), \ldots, \eta(n)=\sigma(n)) \mathbf{P}(\eta(s) \\
& =\sigma(s)=1 \mid \text { under conditions: } \eta(1)=\sigma(1), \ldots, \eta(s-1) \\
& =\sigma(s-1), \eta(s+1)=\sigma(s+1), \eta(n)=\sigma(n)) \\
& \leqslant \mathbf{P}(\eta(1)=\sigma(1), \ldots, \eta(s-1)=\sigma(s-1), \eta(s+1)=\sigma(s+1), \ldots, \eta(n) \\
& =\sigma(n))(1-q)=\mathbf{P}^{\text {mixed }, K^{\prime \prime}}\left(A^{j}\right)
\end{aligned}
$$

because of a conditional probability $\mathbf{P}\left(\eta(s)=1 \mid\right.$ any conditions outside $\left.T_{s}\right) \leqslant 1-q$. Thus, for all these $A^{j}$

$$
\mathbf{P}(\eta(1)=\sigma(1), \ldots, \eta(s)=\sigma(s)=1, \ldots, \eta(n)=\sigma(n)) \leqslant \mathbf{P}^{\text {mixed }, K^{\prime \prime}}\left(A^{j}\right) .
$$

Consider $A^{j}$, such that $\sigma(s)=0$. Now, we use the following trick: together with $A^{j}$ we consider an engaged elementary event $A^{i}$, which is obtained by changing of $\sigma(s)$ into 1 (obviously $A^{i}$ belongs to the same compound event $A(l)$ and for different elementary events $A^{j}$ with $\sigma(s)=0$ we have different elementary events $A^{i}$.

For these two elementary events $A^{j}=[\sigma(1), \ldots, \sigma(s)=0, \ldots, \sigma(n)]$ and $A^{i}=$ $\left[\sigma(1), \ldots, \sigma^{\prime}(s)=1, \ldots, \sigma(n)\right]$ we have

$$
\begin{aligned}
\mathbf{P}(\eta(1)= & \sigma(1), \ldots, \eta(s)=\sigma(s)=0, \ldots, \eta(n)=\sigma(n)) \\
& +\mathbf{P}\left(\eta(1)=\sigma(1), \ldots, \eta(s)=\sigma^{\prime}(s)=1, \ldots, \eta(n)=\sigma(n)\right) \\
= & \mathbf{P}(\eta(1)=\sigma(1), \ldots, \eta(s-1)=\sigma(s-1), \eta(s+1) \\
= & \sigma(s+1), \ldots, \eta(n)=\sigma(n))+\mathbf{P}(\eta(s)
\end{aligned}
$$




$$
\begin{aligned}
& =\sigma(s)=0 \mid \text { under conditions: } \eta(1)=\sigma(1), \ldots, \eta(s-1) \\
& =\sigma(s-1), \eta(s+1) \sigma(s+1), \ldots, \eta(n)=\sigma(n))+\mathbf{P}(\eta(1) \\
& =\sigma(1), \ldots, \eta(s-1)=\sigma(s-1), \eta(s+1) \\
& =\sigma(s+1), \ldots, \eta(n)=\sigma(n)) \mathbf{P}(\eta(s) \\
& =\sigma^{\prime}(s)=1 \mid \text { under conditions: } \eta(1)=\sigma(1), \ldots, \eta(s-1) \\
& =\sigma(s-1), \eta(s+1) \sigma(s+1), \ldots, \eta(n)=\sigma(n))
\end{aligned}
$$

(the following equality is valid due to the fact that the sum of two conditional complementary events is equal to one)

$$
\begin{aligned}
& =\mathbf{P}(\eta(1)=\sigma(1), \ldots, \eta(s-1)=\sigma(s-1), \eta(s+1)=\sigma(s+1), \ldots, \eta(n)=\sigma(n)) \\
& =(q+(1-q)) \mathbf{P}(\eta(1)=\sigma(1), \ldots, \eta(s-1)=\sigma(s-1), \eta(s+1) \\
& =\sigma(s+1), \ldots, \eta(n)=\sigma(n)) \\
& =\mathbf{P}^{\text {mixed }, K^{\prime \prime}}\left(A^{j}\right)+\mathbf{P}^{\text {mixed }, K^{\prime \prime}}\left(A^{i}\right) .
\end{aligned}
$$

Thus, for all $A^{j}$ of the second kind we have

$$
\begin{aligned}
\mathbf{P}(\eta(1)= & \sigma(1), \ldots, \eta(s)=\sigma(s)=0, \ldots, \eta(n)=\sigma(n)) \\
& +\mathbf{P}\left(\eta(1)=\sigma(1), \ldots, \eta(s)=\sigma^{\prime}(s)=1, \ldots, \eta(n)=\sigma(n)\right) \\
= & \mathbf{P}^{\text {mixed }, K^{\prime \prime}}\left(A^{j}\right)+\mathbf{P}^{\text {mixed }, K^{\prime \prime}}\left(A^{i}\right) .
\end{aligned}
$$

In the case when $K^{\prime \prime}=s$ inequality (27) (and therefore Eq. (28)) is proved.

In the general case, when $K^{\prime \prime}$ consists of $l$ numbers (when we "replace" $l$ random variables), we successively repeat $l$ times the same argument and obtain Eq. (28).

Just by putting $K^{\prime \prime}=K$ we complete the proof of the proposition.

The random variables $\xi(k)$ are independent and identically distributed. The mathematical expectation of $\xi(k)$ equals $1-q$.

Now, we show that inequality (26) is valid for the "absolute probability" $P^{\text {abs }}$, that is

$$
P^{\mathrm{abs}}\left(\eta(m)=1 \mid \text { any conditions outside } T_{m}\right) \leqslant 1-q .
$$

Let $\mathbf{P}_{\mathbf{V}}$ be Gibbs measure corresponding to arbitrary boundary conditions and $T_{k}$ be an arbitrary segment. Consider the set of all configurations on the interval $T_{k}$ and the restriction of the measure $\mathbf{P}_{\mathbf{V}}$ on this set. We show that at some value of $l$ the "absolute probability" $P^{\text {abs }}$ that in $T_{k}$ there is at least one regular segment greater than $q>0$ for some constant $q$ not depending on $k$. The event $\eta(m)=1$ means that all segments belonging to $T_{k}$ are nonregular.

Condition (3) and the Peierls argument method directly imply that

$$
P^{\mathrm{abs}}\left(\eta(m)=1 \mid \text { conditions outside } T_{m} \text { are } \varphi^{g r}(x)\right) \leqslant \exp (-\beta t l) .
$$


Now we note that inequality (29) which is just inequality (30) at any conditions outside $T_{k}$ is also held at sufficiently large values of $l$. Indeed, due to Eq. (22) when we increase the value of $l$ the influence of the conditions outside $T_{k}$ on the configuration in $T_{k}$ increases with the rate less than $l$ and therefore at some value of $l$ and for some positive $t_{1}<t$

$$
P^{\mathrm{abs}}\left(\eta(m)=1 \mid \text { any conditions outside } T_{m}\right) \leqslant \exp \left(-\beta t_{1} l\right) \leqslant 1-q .
$$

Now Lemma 8 is a direct consequence of the Strong Law of Large Numbers for $\xi(k)$ and the Proposition. Indeed, consider independent Bernoulli trials when the probability of success at each trial equals $1-q$. According to the Strong Law of Large Numbers, the probability of the event that the density of successes exceeds $1-q^{\prime} ; 0<q^{\prime}<q$, is less than $\frac{1}{4}$, when $V$ tends to infinity. It means that the "absolute probability" of the event that the density of nonsuper-regular segments $T_{k}$ is greater than $1-q^{\prime}$ is less than $\frac{1}{4}$. Due to the proposition, this probability is greater than the $P^{\text {abs }}$ probability of the event that the density of nonsuper-regular segments, $T_{k}$ is greater than $1-q^{\prime}$. In other words, the $P^{\text {abs }}$ probability of the event that the density of super-regular segments $T_{k}$ is less than $1-q^{\prime}$ is less than $\frac{1}{4}$. Thus, the $P^{\text {abs }}$ probability of the event that the density of super-regular segments $T_{k}$ is greater than $1-q^{\prime}$ is greater than $\frac{1}{4}$. Taking into account that each super-regular segment $T_{k}$ contains at least one regular segment, one can see that the last statement implies Lemma 8 if the parameter $p$ is chosen from the open interval $\left(1-q^{\prime} / l, 1\right)$. We choose the value of $p$ as $1-q / 2 l$.

Lemma 8 is proved.

Proof of Lemma 9. Let us consider the set of all long clusters $D_{i}$ with the density of supports less than $p$. Let $\operatorname{supp}(D)=\bigcup_{i=j}^{r} \operatorname{supp}\left(K_{j}\right)$. These supports $K_{i}$ are connected between themselves and with the boundary. Since the density of supports is not greater than $p<1$ the sum of the lengths of bonds in both halves $[-V,-|I| / 2$ and $[|I| / 2, V]$ is not less than $(V-|I| / 2)(1-p)$. When $V$ goes to infinity the sum of lengths of any long cluster with the density less than $p$ tends to infinity, and by condition (22) the impact of these bonds tends to zero.

By choosing the appropriate value of $V$ we complete the proof of Lemma 9. Lemma 9 is proved.

We omit the huge proof of Lemma 5 since it is absolutely analogous to the proof of Lemma 6 . The only difference is the fact that in $\Xi^{1,1,2, \prime \prime}$ overlapped clusters are allowed, so the density of nonregular segments of typical configurations in Lemmas 8, 9 instead of $p$ will be a number less than $1-(1-p)(1-p)$.

Partition functions including only nonlong super clusters satisfy the following key lemma which has a geometrically combinatorial explanation.

\section{Lemma 10.}

$$
\Xi^{1, \prime \prime, 2, l,(n . l .)}=Q \Xi^{1, \prime, 2, \prime \prime,(n . l .)},
$$



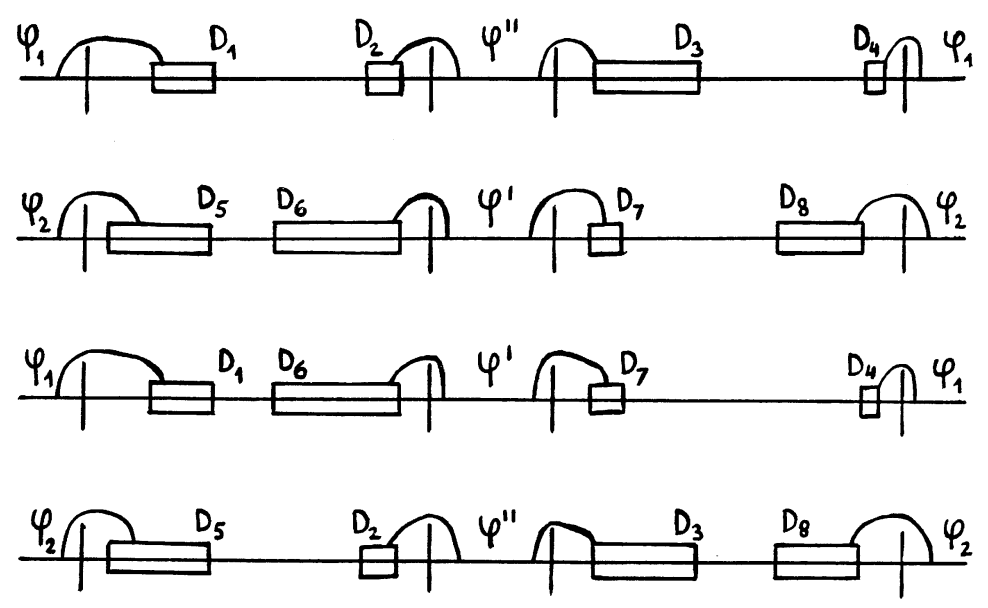

Fig. 1.

where the factor $Q=Q\left(\varphi^{1}(x), \varphi^{2}(x), \varphi^{\prime}(x), \varphi^{\prime \prime}(x)\right)$ is uniformly bounded: $0<$ const $_{1}<$ $Q<$ const $_{2}$.

Remark. The factor $Q$ appears due to the fact that the configurations with minimal energies corresponding to the different boundary conditions do not coincide everywhere (due to Lemma 1 they differ on some finite set and due to condition (2) $Q$ is finite).

Proof. Due to the factor $Q$, without loss of generality, we suppose that the configurations with minimal energies corresponding to the different boundary conditions coincide with $\varphi^{g r}(x)$.

The summations in $\Xi^{1, \prime \prime, 2, l,(n . l .)}=\Xi^{1, l, 2, \prime \prime,(n . l .)}$ are taken over all nonlong, nonordered compatible collections of super clusters.

We put a one-to-one correspondence between the terms of these two super partition functions.

Fig. 1 shows how this one-to-one correspondence can be carried out.

To the term $w\left(D_{1}^{1, \prime \prime}\right) w\left(D_{2}^{1, \prime \prime}\right) w\left(D_{3}^{1, \prime \prime}\right) w\left(D_{4}^{1, \prime \prime}\right) w\left(D_{5}^{2, \prime}\right) w\left(D_{6}^{2, \prime}\right) w\left(D_{7}^{2, \prime}\right) w\left(D_{8}^{2, \prime}\right)$ (the first four factors of this term came from the partition function $\Xi^{1, \prime \prime}$ and the last four factors of this term came from the partition function $\left.\Xi^{2, \prime}\right)$ of the super partition function $\Xi^{1, \prime \prime, 2,,(n . l .)}$ we correspond the term $w\left(D_{1}^{1, \prime}\right) w\left(D_{6}^{1, \prime}\right) w\left(D_{7}^{1, \prime}\right) w\left(D_{4}^{1, \prime}\right) w\left(D_{5}^{2, \prime \prime}\right) w\left(D_{2}^{2, \prime \prime}\right)$ $w\left(D_{3}^{2, \prime \prime}\right) w\left(D_{8}^{2, \prime \prime}\right)$ (the first four factors of this term came from the partition function $\Xi^{1,1}$ and the last four factors of this term came from the partition function $\Xi^{2, \prime \prime}$ ) of the super partition function $\Xi^{1, l, 2,1 \prime,(n . l .)}$.

It can be easily shown that this one-to-one correspondence is well defined: if some term from $\Xi^{1,1,2,1 /,(n . l .)}$ corresponding to the term from $\Xi^{1,1,2, l,(n . l .)}$ does not exist (in other words, the corresponding clusters from $\Xi^{1, \prime}$ or $\Xi^{2, \prime \prime}$ are overlapped) then the term from $\Xi^{1,1 / 2, l,(n . l .)}$ is a long super cluster, which is impossible. The lemma is proved.

Inequality (9) is a direct consequence of Lemma 5 and 10. Lemma 2 is proved. 
Let $\mathbf{P}^{1}$ and $\mathbf{P}^{2}$ be two different extreme Gibbs states of the model (1) corresponding to the boundary conditions $\varphi^{1}(x)$ and $\varphi^{2}(x)$ respectively.

Theorem 2 (Dobrushin [15]). $\mathbf{P}^{1}$ and $\mathbf{P}^{2}$ are singular or coincide.

Proof of Theorem 1. Let $\mathbf{P}^{1}$ and $\mathbf{P}^{2}$ be two different extreme limit Gibbs states of model (1) corresponding to the boundary conditions $\varphi^{1}(x)$ and $\varphi^{2}(x)$, respectively. Due to Lemma $2 \mathbf{P}^{1}$ and $\mathbf{P}^{2}$ are not singular. Therefore, by Theorem $2, \mathbf{P}^{1}$ and $\mathbf{P}^{2}$ coincide, which contradicts the assumption. Theorem 1 is proved.

\section{Conclusions}

In [10], the following conjecture describing sufficient conditions for the absence of phase transitions was formulated:

Conjecture. Any one-dimensional model with discrete (at most countable) spin space and with a unique ground state has a unique Gibbs state if the spin space of this model is finite or the potential of this model is translationally invariant.

Our Theorem 1 is closely related to this conjecture.

The main point in the proof of the uniqueness of Gibbs states is Lemma 10 and the estimation of long super clusters connecting the boundary with the segment $I$. We reduce the summation in $\Xi^{1,1,2, \prime \prime}$ into $\Xi^{1,1,2,1 /,(n . l)}$. It turns out that long clusters from the partition function $\Xi^{1, l, 2, l l,(n . l .)}$ have long interaction elements and therefore are negligible.

Theorem 1 admits generalizations in different directions:

(1) Theorem 1 can be generalized for the models having a unique ground state to within translations. But in this case we have to add one more condition

$$
\mid \sum_{A \subset(-\infty, m] ; B \subset[m, \infty)} U(\varphi(B \cup A)-U(\varphi(B+l \cup A) \mid<\text { const },
$$

where the inequality holds uniformly with respect to the configuration $\varphi(x)$ and integer numbers $m, l$.

(2) Theorem 1 can be generalized for the models with a countable spin space. In this case, condition (3) must be replaced with the following condition:

There exists $\beta^{c r}>0$ such that for all $\beta>\beta^{c r}$ and for any finite set $A \subset \mathbf{Z}^{1}$ with the length $|A|$

$$
\sum_{\varphi^{\prime}(x)} \exp \left(-\beta\left(H\left(\varphi^{\prime}(x)\right)-H\left(\varphi^{g r}(x)\right)\right)\right) \leqslant \exp (-\beta(t|A|)),
$$

where the summation is taken over all possible finite perturbations $\varphi^{\prime}(x)$ of the ground state $\varphi^{g r}$ on the finite set $A$. 
(3) Suppose that in the two-dimensional case the configuration with the minimal energy $\varphi_{V}^{\min , 1}(x) \in \Phi(V)$ differs from the ground state on some set of finite volume $C$, where the constant $C$ can be chosen uniformly with respect to $V$ and boundary conditions $\varphi^{1}(x)$. Then by using the method of this paper we can also prove the uniqueness theorem for two-dimensional models with a unique ground state (see also [16] but only at "very" low temperatures (in one dimension the condition on low temperatures comes just from the need in condition (3)). In fact, even if condition (3) is held, in two-dimension the phenomenon of percolation does not allow us to prove the analog of Lemma 7 at any temperature (in one-dimension the constant $p$ in Lemma 8 can be an arbitrary number, less than one, but in two-dimension in order to resist a percolation, $p$ must be bounded above, say by 0.503 ).

\section{Acknowledgements}

The author thanks the referee for his or her useful suggestions.

\section{References}

[1] R.L. Dobrushin, Teor. Veroyat. Primenenie. 18 (2) (1968) 201-229.

[2] R.L. Dobrushin, Funk. Anal. Pril. 2 (4) (1968) 44-57.

[3] D. Ruelle, Commun. Math. Phys. 9 (1968) 267-278.

[4] F.J. Dyson, Comm. Math. Phys. 12 (1969) 91-107.

[5] F. Spitzer, J. Func. Anal. 20 (1975) 240-255.

[6] S. Kalikow, Ann. Prob. 5 (1977) 467-469.

[7] W.G. Sullivan, Comm. Dublin Inst. Adv. Studies (1975).

[8] M.J. Miyamoto, Math. Kyoto Univ. 24 (4) (1984) 679-688.

[9] A.A. Kerimov, J. Stat. Phys. 72 (3/4) (1993) 571-620.

[10] A.A. Kerimov, Physica A 225 (1996) 271-276.

[11] H. Berbee, Ann. Probab. 17 (4) (1989) 1416-1431.

[12] J. Frohlich, T. Spencer, Comm. Math. Phys. 84 (1) (1982) 87-101.

[13] M. Aizenman, J.T. Chayes, L. Chayes, C.M. Newman, J. Stat. Phys. 50 (1/2) (1988) 1-40.

[14] J. Bricmont, K. Kuroda, J.L. Lebowitz, Commun. Math. Phys. 101 (1985) 501-538.

[15] R.L. Dobrushin, Funk. Anal. Pril. 22 (1974) 31-43.

[16] J.L. Lebowitz, A. Mazel, Comm. Math. Phys. 189 (1997) 311-321. 